\title{
Amentoflavone: A Bifunctional Metal Chelator that Controls the Formation of Neurotoxic Soluble A $\boldsymbol{\beta}_{42}$ Oligomers
}

Liang Sun, ${ }^{a}$ Anuj K. Sharma, ${ }^{\mathrm{b}}$ Byung-Hee Han, ${ }^{\mathrm{c}}$ Liviu M. Mirica ${ }^{\mathrm{a}} \mathrm{d}, *$

${ }^{a}$ Department of Chemistry, University of Illinois at Urbana-Champaign, 600 S. Mathews Avenue, Urbana, Illinois 61801, USA

${ }^{b}$ Department of Chemistry, Central University of Rajasthan, Bandarsindari, Dist. Ajmer-305801, Rajasthan, India

${ }^{\mathrm{c}}$ Department of Pharmacology, A.T. Still University of Health Sciences, Kirksville, MO 63501, USA

${ }^{\mathrm{d}}$ Hope Center for Neurological Disorders, Washington University School of Medicine, St. Louis, Missouri 63110, USA

*Correspondence: mirica@illinois.edu (L.M.M.) 


\section{Table of Contents}

1. UV-vis spectrum of amentoflavone (AMF) S3

2. Fluorescence spectrum of AMF S3

3. Possible metal binding modes of AMF S4

4. Fluorescence microscopy images of AD mice brain sections $\quad$ S4

5. Autofluorescence images of AD mice brain sections $\quad \mathrm{S} 4$

6. Additional docking poses of AMF to various $A \beta$ aggregates $\quad$ S5

7. Docking scores and Glide e-model energies for all docking poses S6

8. TEM images for $\mathrm{Zn}^{2+}-\mathrm{A} \beta_{42}$ inhibition and disaggregation studies $\quad \mathrm{S} 7$ 


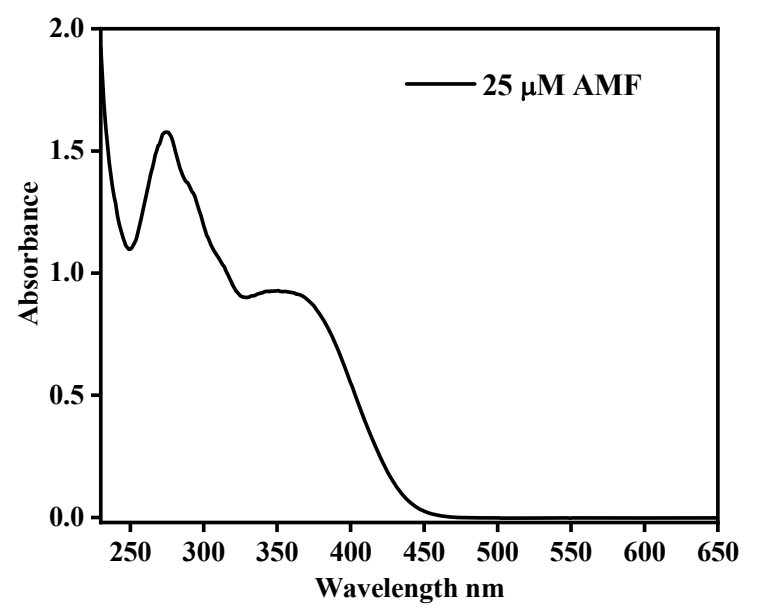

Figure S1. UV-vis spectrum of amentoflavone (AMF, $25 \mu \mathrm{M})$ in PBS.

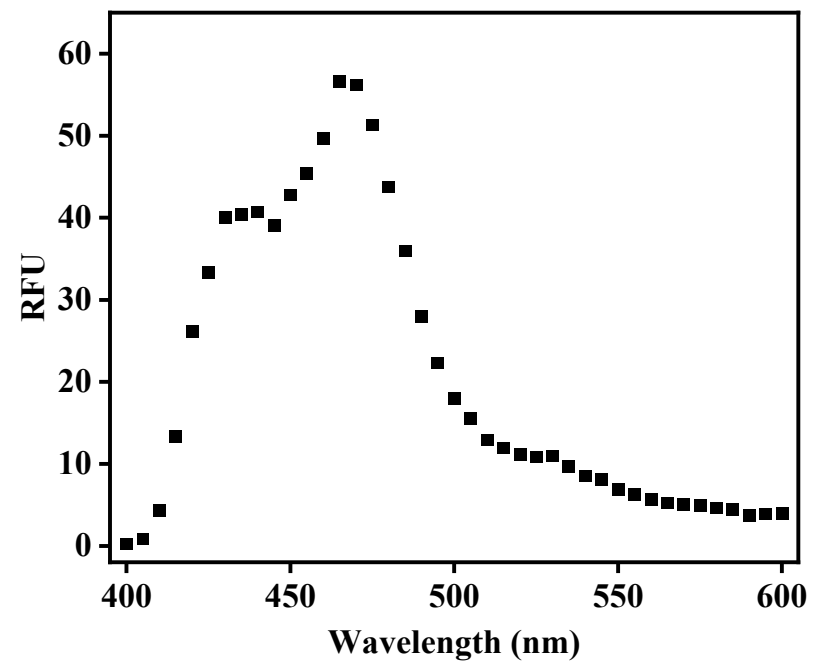

Figure S2. Fluorescence spectrum of AMF $(50 \mu \mathrm{M})$ in $\operatorname{PBS}\left(\lambda_{\mathrm{ex}}=310 \mathrm{~nm}\right)$. 
<smiles></smiles><smiles>[M]OCc1cc(O)cc2oc(-c3ccc(O[M])c(-c4c(O[M])cc(O)c5c(=O)cc(-c6ccc(O)cc6)oc45)c3)cc(=O)c12</smiles>

Figure S3. Proposed binding modes of metal ions to AMF. The adjacent ketone and phenolate groups, or the adjacent phenolate groups, are proposed to chelate the metal ions.
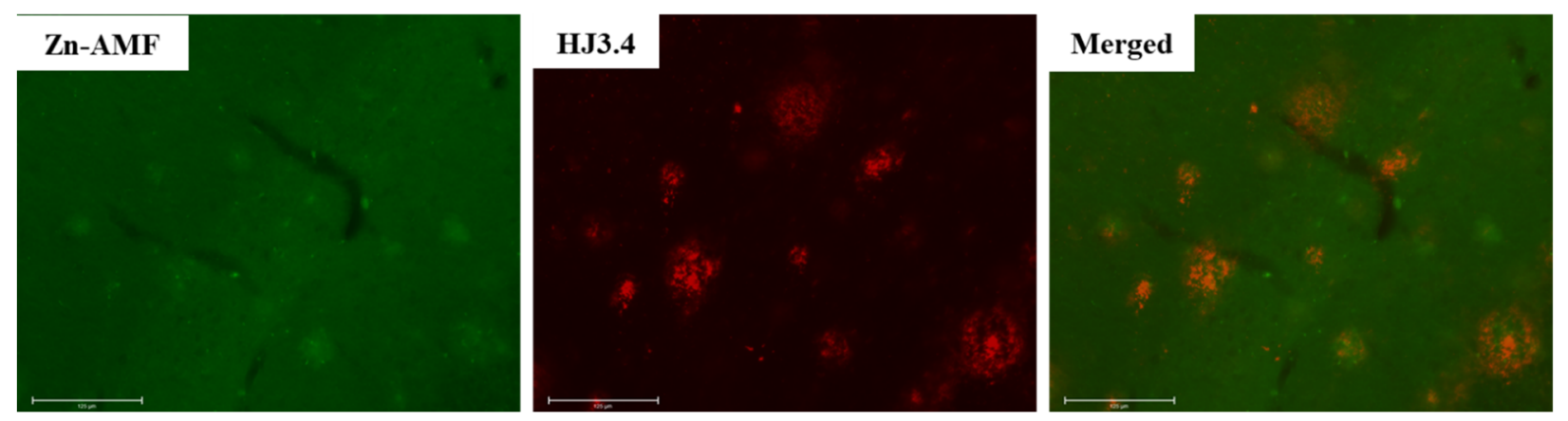

Figure S4. Fluorescence microscopy images of $5 \times$ FAD mouse brain sections coincubated with $\mathrm{Zn}^{2+}$-AMF (left panel) - showing minimal staining, the AF594-HJ3.4 antibody (middle panel), and merged images (right panel). Concentrations used: $[\mathrm{AMF}]=\left[\mathrm{Zn}^{2+}\right]=25 \mu \mathrm{M},[\mathrm{HJ} 3.4]=1$ $\mu \mathrm{g} / \mathrm{ml}$ (scale bar: $125 \mu \mathrm{m}$ ).
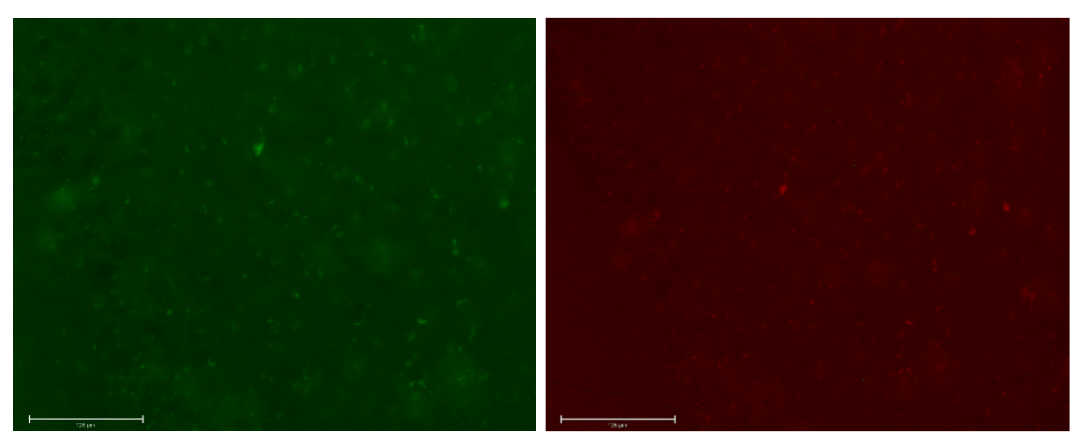

Figure S5. Autofluorescence images of $5 \times$ FAD mouse brain sections in the FITC channel (left panel) and Texas Red channel (right panel). 
(a)
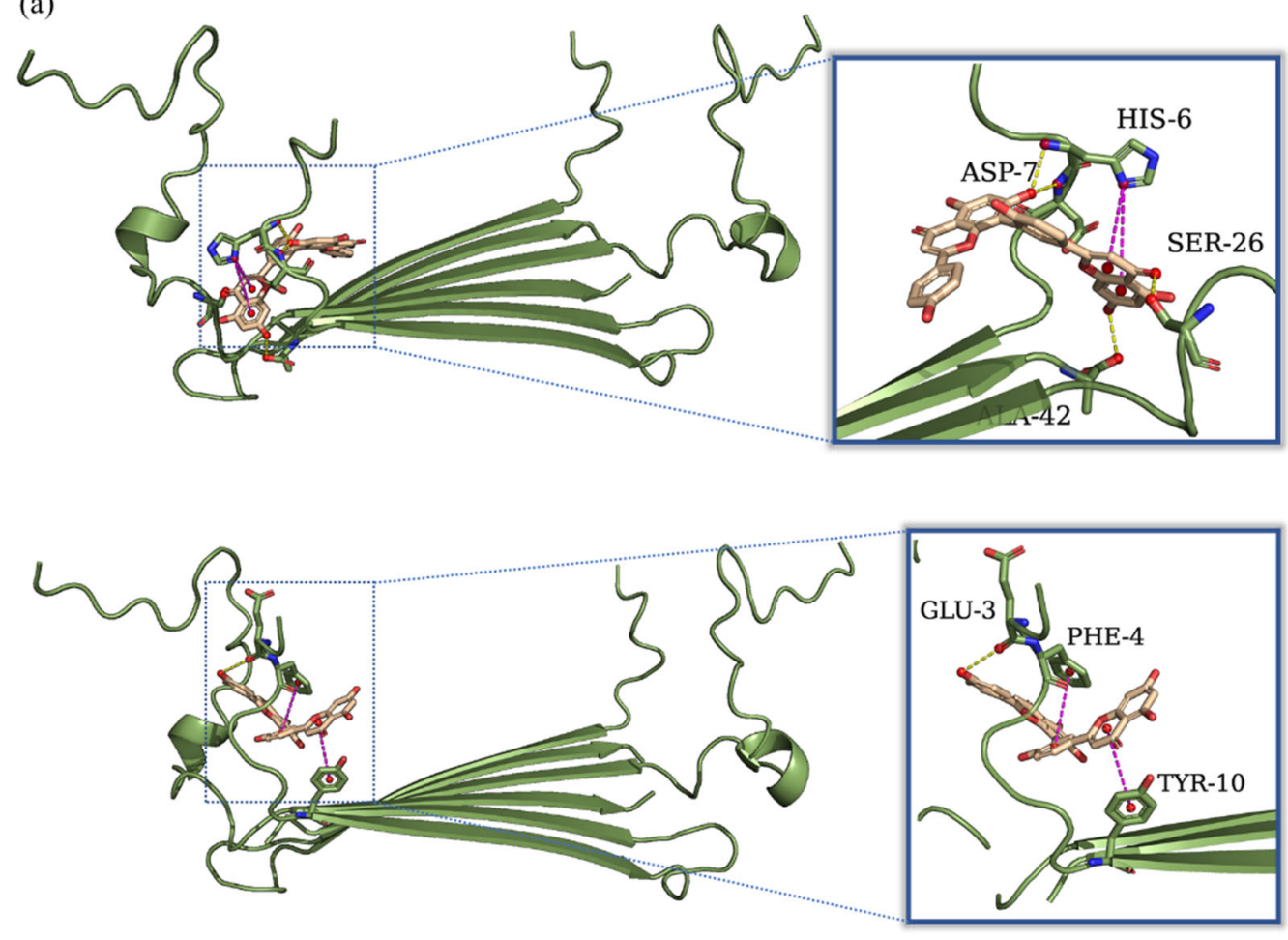

(b)

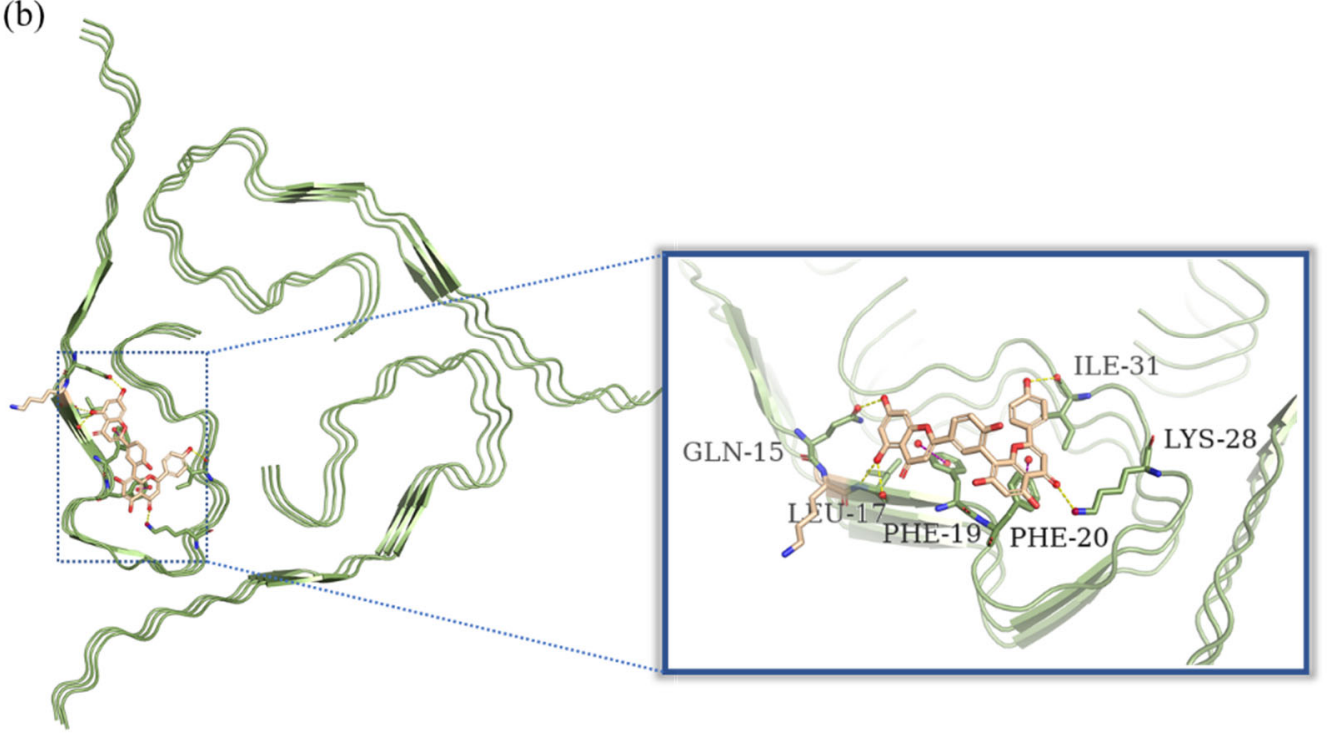

Figure S6. (a) Other docking poses of AMF to $A \beta_{42}$ oligomers (PDB ID:6RHY). The top figure shows that AMF has strong interaction with His-6 and Asp-7 through $\pi$-cation interaction and hydrogen bond. The bottom figure shows AMF binds to the oligomers with Glu-3 with hydrogen bonds and Phe-4, Tyr- 10 with $\pi-\pi$ interactions. (b) Additional docking pose of AMF to A $\beta_{40}$ fibrils (PDB ID: 2M4J) with the second highest glide e-model energy $(-64.47 \mathrm{kcal} / \mathrm{mol})$. This figure shows AMF interacts with the KLVFF region via $\pi-\pi$ interactions (Phe-19, Phe-20) and hydrogen bonds (Leu-17, Lys-28, Ile-31). The predicted hydrogen bonds and $\pi-\pi$ interactions are highlighted with yellow and magenta dashed lines. 
Table S1. Docking score and Glide e-model energy for each PDB structure corresponding to $A \beta_{42}$ oligomers, $A \beta_{40}$ fibrils, and $A \beta_{42}$ fibrils, respectively.

\begin{tabular}{|cc|}
\hline \multicolumn{2}{|c|}{ A $\beta_{42}$ oligomers (PDB ID: 6RHY) } \\
Docking score & Glide e-model(kcal/mol) \\
\hline $\mathbf{- 4 . 4 8 3}$ & $\mathbf{- 5 8 . 9 4 0}$ \\
$\mathbf{- 4 . 4 6 5}$ & $\mathbf{- 6 5 . 5 0 8}$ \\
-2.425 & $\mathbf{- 6 6 . 0 4 9}$ \\
-2.280 & $\mathbf{- 5 9 . 2 9 2}$ \\
\hline
\end{tabular}

\begin{tabular}{|c|c|}
\hline \multicolumn{2}{|c|}{ A $\beta_{40}$ fibrils (PDB ID: 2M4J) } \\
\hline Docking score & Glide e-model(kcal/mol) \\
\hline-5.198 & -60.723 \\
\hline-4.795 & -67.033 \\
\hline-3.677 & -64.446 \\
\hline-1.244 & -49.334 \\
\hline
\end{tabular}

\begin{tabular}{|cc|}
\hline \multicolumn{2}{|c|}{ A $\beta_{42}$ fibrils (PDB ID: 5OQV) } \\
Docking score & Glide e-model(kcal/mol) \\
\hline $\mathbf{- 6 . 5 5 7}$ & $\mathbf{- 6 7 . 9 8 6}$ \\
$\mathbf{- 5 . 2 3 4}$ & $\mathbf{- 7 2 . 9 7 7}$ \\
-3.219 & -65.607 \\
-2.927 & -64.206 \\
\hline
\end{tabular}



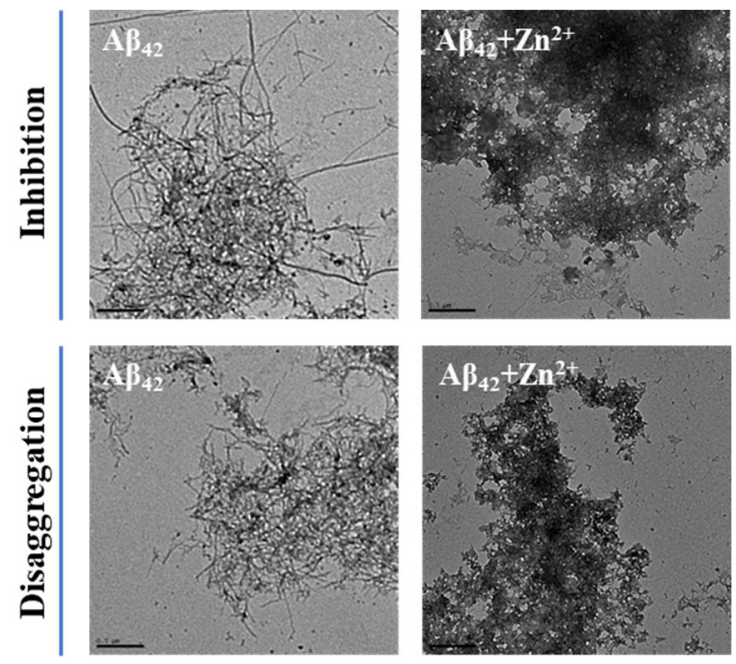

Figure S7. Additional TEM images for the inhibition of $A \beta_{42}$ aggregation and disaggregation of $\mathrm{A} \beta_{42}$ fibrils, in the absence or presence $\mathrm{Zn}^{2+}$ ions. 